\title{
A CALCULUS CHOLECYSTITIS PERFORATING AT THE NECK
}

Jayashri Pandya ${ }^{1}$, Sudatta Waghmare ${ }^{2}$

\section{HOW TO CITE THIS ARTICLE:}

Jayashri Pandya, Sudatta Waghmare. "A Calculus Cholecystitis Perforating at the Neck". Journal of Evolution of Medical and Dental Sciences 2014; Vol. 3, Issue 39, August 28; Page: 9956-9958,

DOI: $10.14260 /$ jemds $/ 2014 / 3290$

ABSTRACT: Gallbladder perforation is a rare complication of cholecystitis and cholelithiasis. The high morbidity and mortality rates associated with this condition are due to delays in diagnosis and treatment since signs and symptoms of perforation do not differ significantly from those of uncomplicated cholecystitis. We report a case of 17 year old boy who presented with perforation at the neck of gall bladder with extrusion of a stone. It was associated with bile infected with S. typhi.

KEYWORDS: Gall bladder perforation, cholecystitis, cholelithiasis.

INTRODUCTION: Spontaneous perforation of the gallbladder is rare. It occurs in approximately 3\% of cases of acute cholecystitis, usually associated with advanced age and the presence of stones ${ }^{1}$. Fundus is the commonest site of perforation. Incidence of perforation is lower with acalculus cholecystitis ${ }^{2}$.Rare cause of GB perforation is typhoid fever, where patients carry Salmonella typhi in the GB and develop acute acalculus cholecystitis.

We report a case of 17 year old boy who presented with perforation at the neck of gall bladder with extrusion of a stone. It was associated with bile infected with S. typhi.

CASE PRESENTATION: A 17 year old male presented with history of pain in right upper quadrant of abdomen since 2 days. It was associated with high-grade continuous fever not subsiding after medication. There was mild tachycardia. Abdominal examination showed tenderness in right hypochondriac region.

The laboratory tests showed leukocytosis (with neutrophilia). Abdominal x-ray was normal. Ultrasonography of abdomen showed distended Gall Bladder with mild thickening and cholelithiasis with the possibility of perforation. A contrast enhanced CT scan of abdomen revealed impacted calculus of $1 \mathrm{~cm}$ at the neck with one extruded stone with a small pericholecystic collection likely to be a sealed off gall bladder perforation. (Figure 1).

In view of these findings it was decided to explore the patient. Intraoperatively, the omentum, colon and duodenum were adherent to the gall bladder. After adhesiolysis, a $0.5 \times 0.5 \mathrm{~cm}$ perforation was seen at the neck of the gall bladder with necrotic edges and extruded stone with minimal purulent collection (Figure 2). Bile admixed with pus was sent for culture. Cholecystectomy was performed. Postoperative period was uneventful.

The histopathology report of the specimen showed features of acute cholecystitis. The bile culture revealed S. typhi. Blood, urine cultures were negative for S. typhi.

DISCUSSION: Perforation of the gall bladder may occur in both calculus and acalculus cholecystitis. In cholelithiasis, the sequence of events that leads to acute cholecystitis and subsequently to perforation is thought to result from occlusion of the cystic duct by a calculus, resulting in retention of intraluminal secretions. Distension of the organ with a consequent rise in intraluminal pressure 
impedes venous and lymphatic drainage, leading to vascular compromise and ultimately to necrosis and perforation of the wall of the gallbladder ${ }^{3}$. In the absence of stone disease, typhoid fever is also known to occasionally cause spontaneous perforation of the gall bladder ${ }^{4}$. In such cases perforation is thought to result from intense inflammation leading to thrombosis of the blood vessels.

The presumed cause of perforation in this case was impacted stone in the neck causing vascular compromise and pressure necrosis of the gall bladder wall. The presence of S. typhi may have compounded the problem and accelerated the process, as the perforation was found at the neck of the gall bladder which is a far less common site than the fundus (due to its poorer vascularity). ${ }^{3}$

Epidemiological studies conducted in endemic regions have indicated that there is a strong link between the development of the chronic carrier state and the presence of gallstones. In fact, approximately $90 \%$ of chronically infected carriers have gallstones. ${ }^{5}$ However, whether these microorganisms have a primary role in causing cholecystitis or cholelithiasis, or are merely colonizers of a previously damaged gall bladder, is still unknown.

Although management is same, it is difficult to determine whether the patient developed gallstones first and then became infected with $S$ typhi, or vice versa.

Niemeier classified gallbladder perforation into three clinical types: type 1 (acute) presentation as generalized peritonitis; type 2 (subacute) - pericholecystic abscess and localized peritonitis; type 3 (chronic) - cholecystoenteric fistula. ${ }^{6}$ This patient had a type 2 perforation.

It is difficult to diagnose perforation of gall bladder clinically. Ultrasonography and computerized tomography (CT) may demonstrate abdominal fluid but lack specificity to diagnose gall bladder perforation. ${ }^{7}$

Kim et al in their comparative study of CT and ultrasonography with gallbladder perforation detected the site of perforation in $50 \%$ of patients on CT but in no patient on ultrasonography ${ }^{8}$. Sood et al noted that the sonographic hole sign, in which the defect in GB wall is visualized, is the only reliable sign of gallbladder perforation. ${ }^{9}$

The best form of management is early surgery as delay in surgery increases both mortality and morbidity of patient. ${ }^{10}$

CONCLUSION: Although GB perforation due to stones is predominantly found in the elderly, it can occur in the young, so high index of suspicion in acute upper abdomen is required. Early diagnosis of gallbladder perforation and timely intervention are of crucial importance. Ultrasound scan and CT scan compliment the clinical diagnosis. "What the mind does not think, the eyes do not see" This dictum should be remembered.

\section{REFERENCES:}

1. Simmons TC, Miller C, Weaver R. Spontaneous gallbladder perforation. Am Surg 1989; 55: 311-3.

2. Chalupa P, Kaspar M, Holub M. Acute acalculous cholecystitis with pericholecystitis in a patient with Epstein-Barr Virus infectious mononucleosis. Med Sci Monit 2009, 15: CS30-33.

3. Saad Akhtar Khan, Gulfam, Abdul Wahid Anwer, Zeeshan Arshad, Khalid Hameed, Mustafa Shoaib. Gall bladder perforation: A rare complication of Acute Cholecystitis. J Pak Med Assoc, Vol. 60, No. 3, March 2010: 228.

4. Ugwu BT, Yiltok SJ, Kidmas AT, Opaluwa AS. Typhoid Intestinal perforation in north central Nigeria. West Afr J Med 2005; 24: 1-6. 


\section{CASE REPORT}

5. Khatri N, S, Maskey P, Poudel S, Jaiswal V, K., Karkey A, Koirala S, Shakya N, Agrawal K, Arjyal A, Day J et al. Evidence That Gall Bladder Carriage of Salmonella Paratyphi A Maybe an Important Factor in the Increasing Incidence of This Infection in South Asia. Ann Int Med 2009Apr 21; 150 (8): 567-8.

6. Neimeier OW. Acute Free Perforation of Gallbladder. Ann Surg 1934; 99: 922-44.

7. Pandey A, Gangopadhyay AN, Kumar V. Gall bladder perforation as a complication of typhoid fever. Saudi J Gastroenterol 2008; 14: 213.

8. Kim PN, Lee KS, Kim IY, Bae WK, Lee BH. Gallbladder perforation: comparison of US findings with CT. Abdomen Imaging 1994; 19: 239-42.

9. Sood BP, Kalra N, Gupta S, Sidhu R, Gulati M, Khandelwal N, Suri S. Role of sonography in the diagnosis of gallbladder perforation. J Clin Ultrasound 2002; 30: 270-274.

10. Menakuru SR, Kaman L, Behera A, Singh R, Katariya RN. Current management of gall bladder perforations. ANZ J Surg 2004; 74: 843-846.

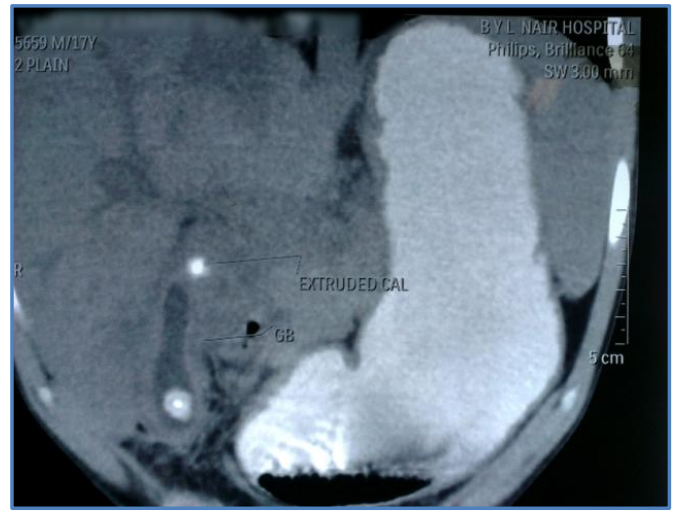

Fig. 1

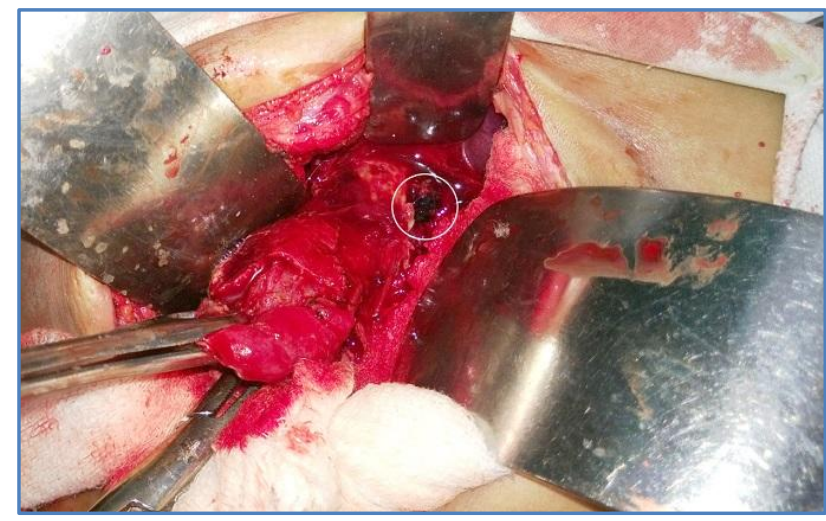

Fig. 2

\section{AUTHORS:}

1. Jayashri Pandya

2. Sudatta Waghmare

\section{PARTICULARS OF CONTRIBUTORS:}

1. Professor, Department of General Surgery, TNMC and BYL Nair Hospital, Mumbai.

2. Assistant Professor, Department of General Surgery, TNMC and BYL Nair Hospital, Mumbai.

\section{NAME ADDRESS EMAIL ID OF THE} CORRESPONDING AUTHOR:

Dr. Jayashri Pandya,

B/101, Gokul Monarch,

Thakur Complex, Kandivali East,

Mumbai-400101.

Email: smruti63@hotmail.com

Date of Submission: 11/08/2014.

Date of Peer Review: 12/08/2014.

Date of Acceptance: 21/08/2014.

Date of Publishing: 28/08/2014. 